\title{
Development of Alarm System Based on Speech Recognition Qingsong Zhu ${ }^{1}$, Hongzhao $\mathrm{Li}^{1}$, Ruyan $\mathrm{Ye}^{1}$, Meide De ${ }^{1}$, Fayue Zheng ${ }^{1}$ ${ }^{1}$ Beijing Polytechnic, Beijing 100176
}

Keywords: Speech recognition; Alarm system; Real-time monitoring; Protection of residents; Security

\begin{abstract}
Aiming at the burglary, fire and the safety and health of the elderly in current living quarters, a kind of alarm system based on speech recognition is developed. The video monitoring device can be triggered by the voice alarm signal which is sent to the mobile client at the same time. Monitor people can see the scene immediately and take measures to help people in danger. It is proved that the speech recognition rate can achieve alarm and start monitoring in $0.5 \mathrm{~s}$ and reach more than $98 \%$ speech recognition in an environment of no more than $50 \mathrm{~dB}$, which can reduce the personal injury and property damage due to unexpected events with no alarming in time. The system has a positive significance in the protection of the residents to a certain extent.
\end{abstract}

\section{Introduction}

With the continuous improvement of living standards in recent years, people's security awareness is also growing. It is a urgently problem to be solved that how to ensure the safety of residents to the maximum extent and get the alarm information and go to the spot to lift the danger in time when the resident's security is violated. Some criminal's burglary, robbery, murder and other crimes can be seen on the internet. Although the aggrieved person let out cry, struggled to resist, but all these were not able to call to rescue. It was too late when found. Or the family suddenly found fire, smoke filled with the air at night, but the party had lost himself (herself) and can't call the police. Or the elderly suddenly fell ill, they can't move with no phone and were far away from the phone, which lead to tragedy.

All of these show that it is necessary to find a new convenient and reliable alarm mode.

Voice alarm does not require contact and just needs a few words. According to the content of the collected speech signal, the alarm system analyzes and judges the alarm type and sent it to family or the district control center, so the family or community management center according to the alarm information to take appropriate rescue operations. Therefore voice alarm has a unique advantage [1-5].

At present, the development of speech recognition technology has been more mature [6-8], the recognition accuracy of speech recognition system for small vocabulary is more than $99 \%[9,10]$. In addition, speech recognition chip with low price and high performance has laid a solid foundation for the application of speech recognition technology.

From the application technology distribution situation analysis of domestic and foreign patent, the patent concentrated distribution in the feature extraction of speech recognition, model building, recognition algorithm and language processing technology. But the speech recognition technology used in the field of security is very small.

In summary, the voice alarm has a wide range of application value. In this paper a kind of speech recognition alarm system is designed. By using this system people can monitor the scene and take measures to minimize the loss caused by the sudden crisis in area residents.

\section{System Diagram}

In Fig. 1, the function of voice input device is to collect the voice information and carry out the content identification. Speech recognition device will recognize the voice alarm information and prior stored alarm segment to match. Voice alarm information will be stored in storing device. When the recognition of the speech alarm information is matched with the prior stored alarm, the 
type of alarm will be determined and alarm mode will be started. Alarm information will be sent to the remote sever then to the receiving terminal. Preset receiving terminal can be a receiving device of a residential property or mobile phone of relatives or third party receiving device. Voice input device 1 is microphone. Monitoring device is a rotational camera installed on the wall to avoid occlusion. Receiving terminal is cellphone.

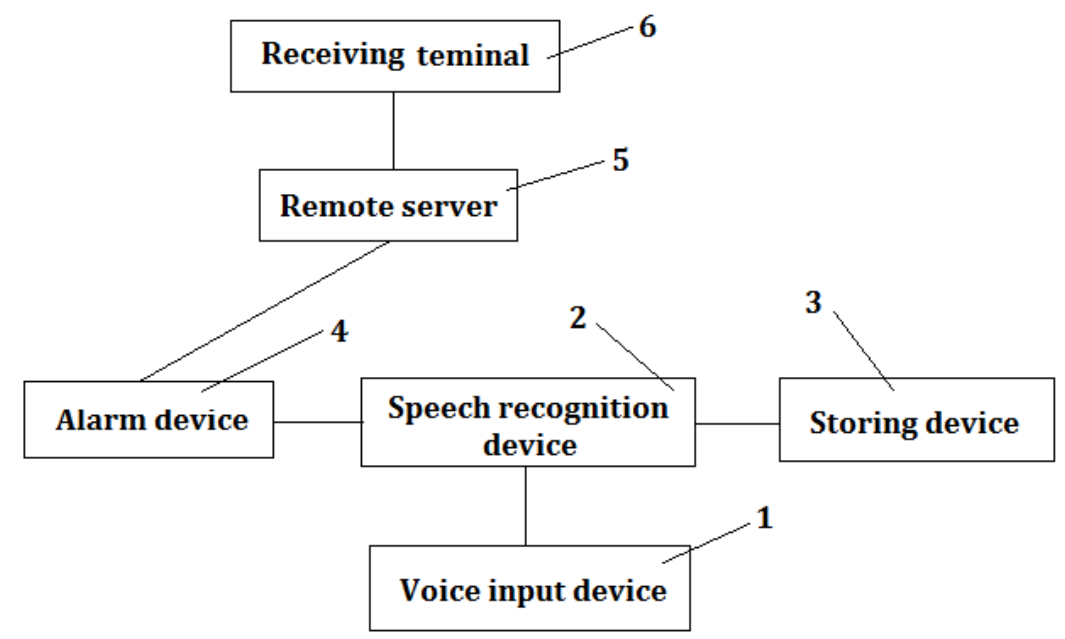

Figure 1. Speech recognition alarm system diagram

User must complete the preset before using alarm system. Preset alarm information depends on the user. User can set some words that are not commonly used to express specific alarm information. Such as preset "red star "mean "burglary "and the alarm type is "police", when users call out "red star", the voice alarm information will be identified as "burglary" and sent to receiving terminal 6.Once the receiving terminal 6 receives the alarm information, it will deal with the alarm signal and display the corresponding alarm content. Alarm content can be text or sound. At the same time it will start the video monitor device and the third party who has received the alarm can open the video monitor device and control operation to see everything happening in the room. Of course the third party can choose can decide whether to open the video monitor device. All the information including the video will be stored in remote sever in order to record and query.

By this method, the accuracy of the alarm can be improved. Since the third party is often a relative, he/she will immediately take all the way to the police, which improve the effectiveness of the alarm and protect life and property safety.

\section{Hard and Software Implementation}

Hard Implementation Hardware frame diagram is shown in Fig.2. Voice information is collected by microphone and then sent to voice chip LD3320 which can store 50 no more than 10 Chinese characters. A parallel communication is adopted between the voice chip and the processor.C8051 is selected as the processor. If there is matching voice information, the processor receives it and sends it to communication module MU509 and send a text message to the default mobile phone. MU509 is $3 \mathrm{G}$ module of China Unicom, which supports SMS and 3G communications. At the same time the rotational station's power is connected and the rotational station is started.Starcam T7837WIP produced by WEISIDAKANG is selected as the rotational station. It can connect the center computer by netting twine or connect the route by WIFI.Route connect the center computer which can save the audio and video in the TF card.The voice and video information can be monitored by center computer. The remote mobile phone can implement monitoring by being connected through the network platform.

Speech recognition device is composed of a processor and a voice chip. The processor is used to initialize the voice chip and previously save the alarm speech information. The voice chip is based on the non-specific human speech recognition technology. It integrates a high precision A/D and D/A interface, which can realize voice recognition / voice / man-machine conversation function and 
is no longer required for external Flash and RAM. It is used to recognize the alarm speech information and compare with the previously stored alarm segment in order to determine the alarm type and start the alarm mode.

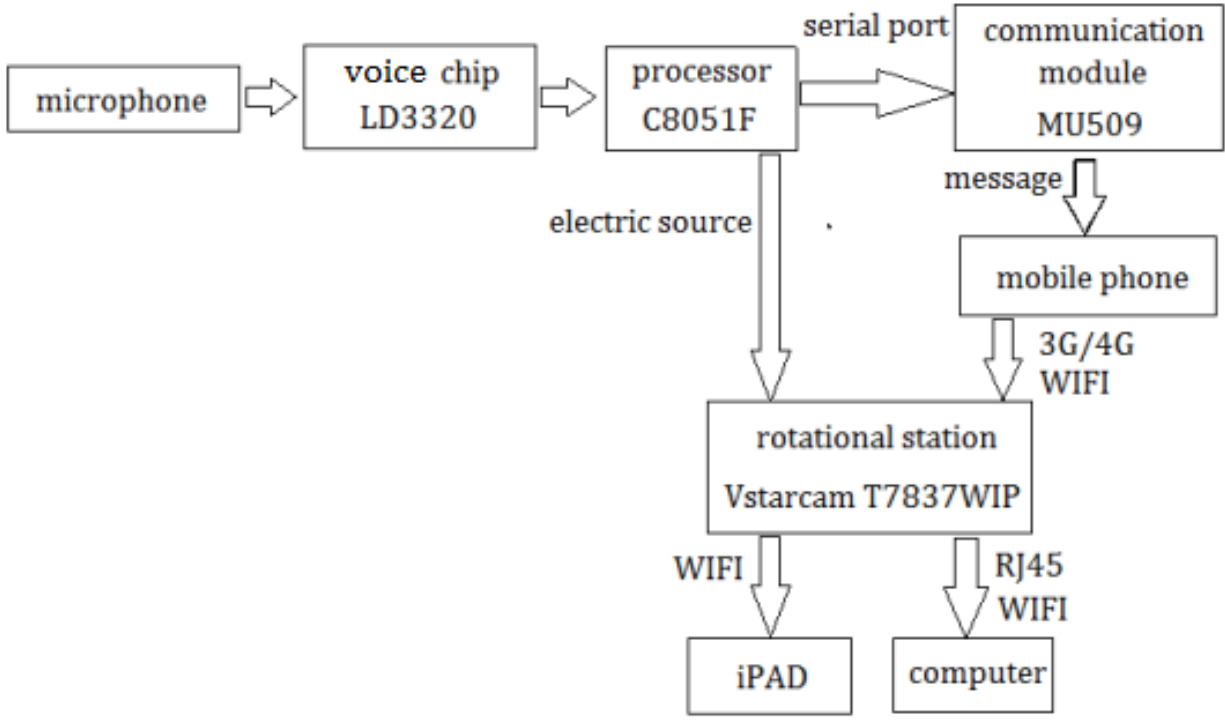

Figure 2. Hardware frame diagram
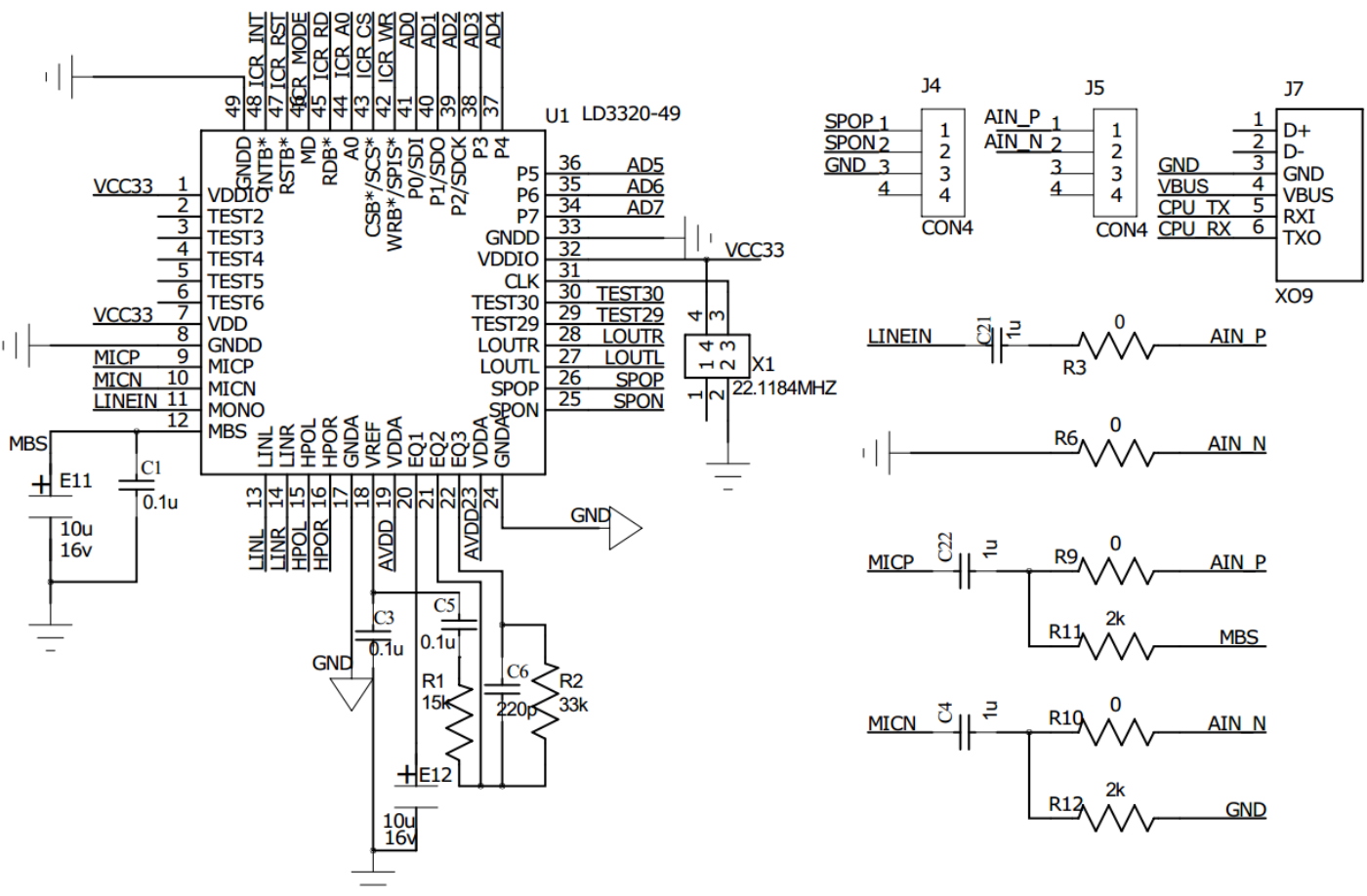

Figure 3. Speech recognition and alarm circuit

Electric source is $12 \mathrm{~V}$ input.LM1117-3.3 conversion 3.3V; LM2596-5V conversion 5V and LM2596ADJ conversion $3.8 \mathrm{~V}$ are supplied for processor voice chip, rotational station and communication module respectively.

The circuit diagram of the system is shown in Fig. 3-4. Fig.4 is the speech recognition and alarm circuit.Fig. 5 is the circuit of controlling system.

\section{Software Implementation}

The monitoring software interface of the system is shown in Fig.6.The interface of the software can be single screen display and can also be divided into multiple screen display. The user can choose 
the corresponding processing according to the operation button of the lower right corner of the software interface.

\section{Experiment and Result}

Testing environment: Living room $\left(25 \mathrm{~m}^{2}\right)$ of an ordinary house with three rooms $\left(100 \mathrm{~m}^{2}\right)$.

Background noise level: $\leqslant 50 \mathrm{~dB}$

Test method and process:

Voice template recording: 3 kinds of voice templates are recorded saved. Input the "I want to the hospital", "Fire!", "Help! "As the three kinds of voice templates.

(1)Preset the mobile phone that can receive messages.

(2)Install the APP on the cellphone, which can control the rotational station. Place a server in the other room.

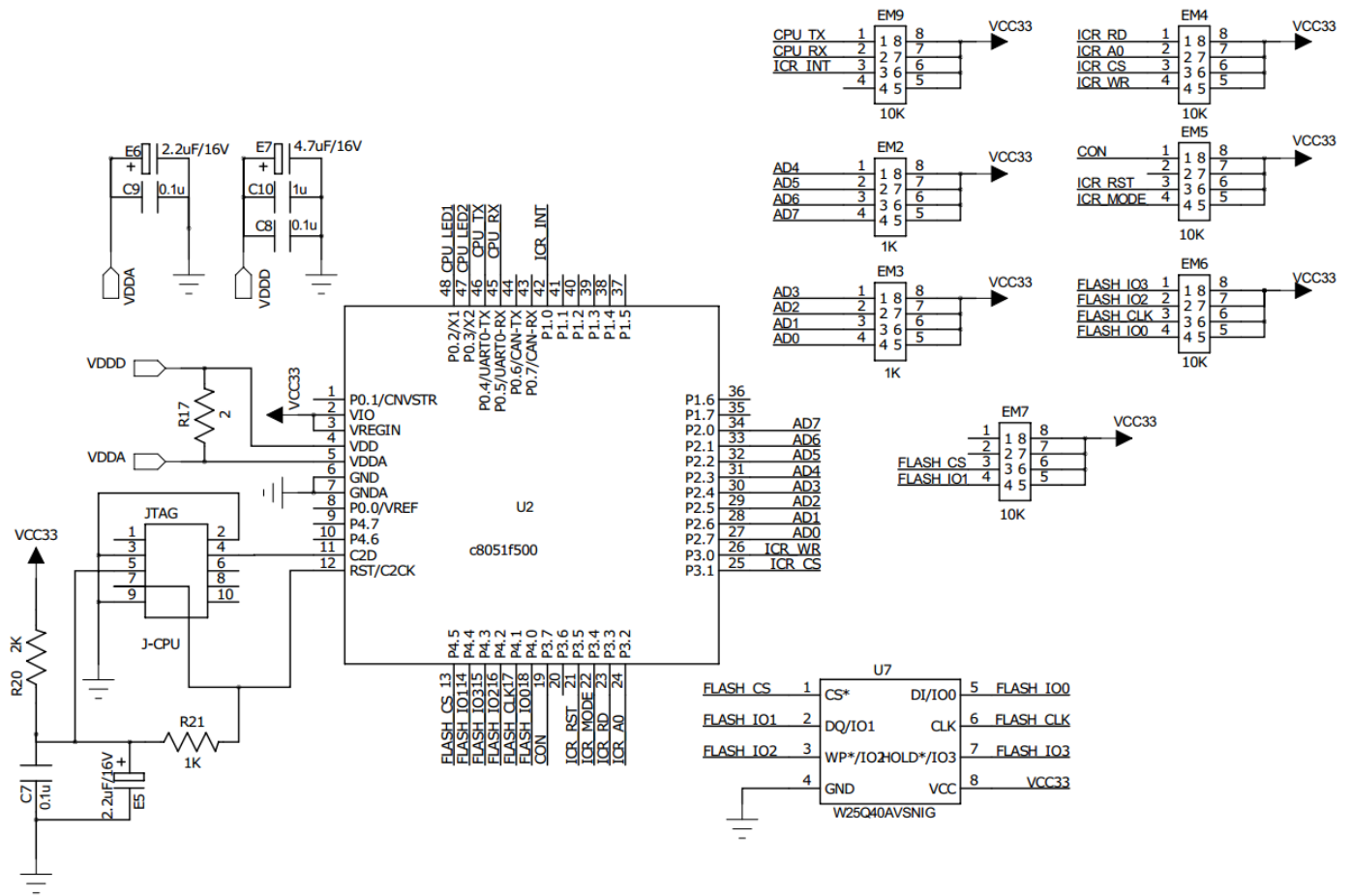

Figure 4. Circuit of controlling system

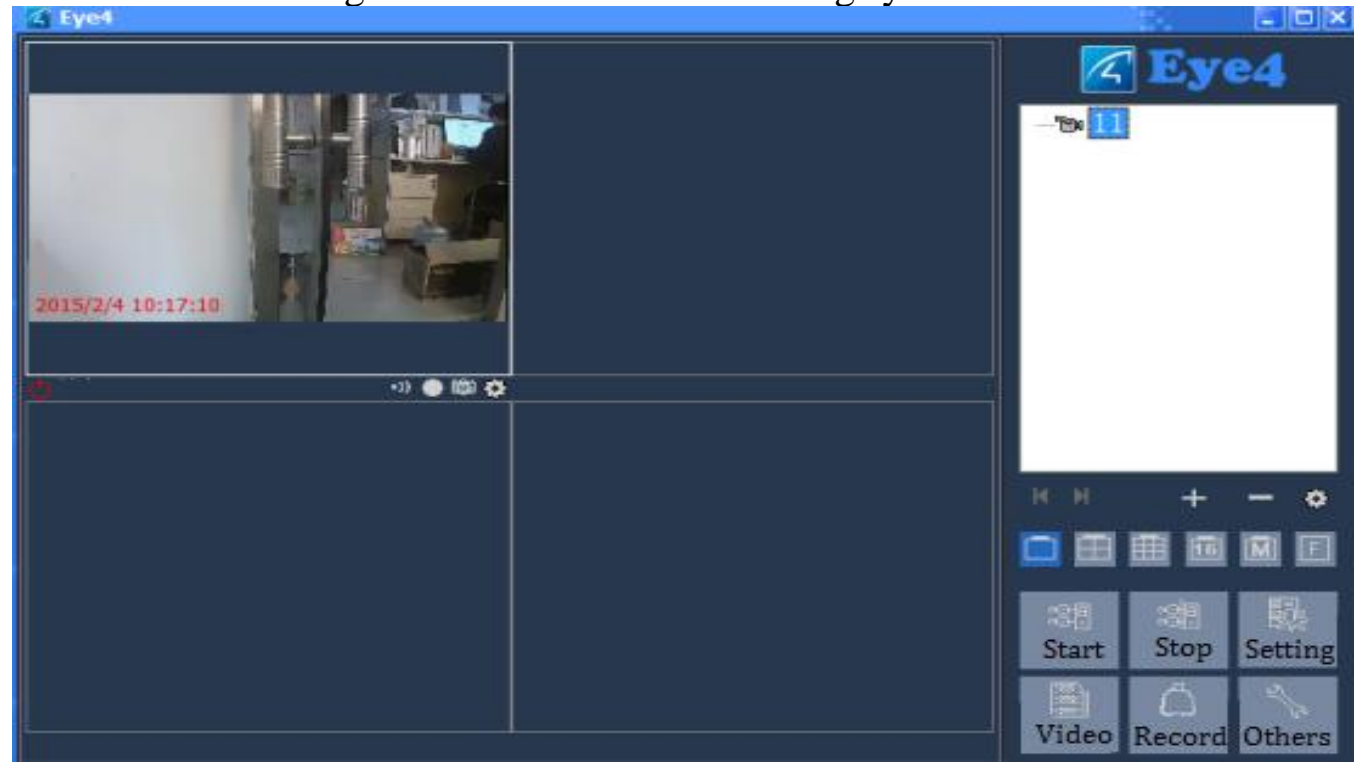

Figure 5. System monitoring software interface

(3)Mounting camera and system control box.

(4)Connect devices and ensure the normal operation. 
(5)One person in the living room alarms, another holds a mobile phone in the outdoor receiving alarm information. Alarm 100 times with different kind.

Result: Alarm recognition rate can reach $98 \%$ within the effective range of $10 \mathrm{~m}$.

\section{Conclusion}

The speech recognition alarm device is convenient and reliable and the cost is low.It can be applied in Intelligent Community widely. But because the voice is easy to be disturbed by the background noise, it is easy to cause false alarm when the room is very noisy.

\section{Acknowledgement}

This study funded by the collaborative innovation project in Chaoyang District of Beijing "Development of Alarm System Based on Speech Recognition". The project number is XC1309.

\section{References}

[1] Jie Chen. Design of alarm system based on speech recognition and its application in broadcasting [J]. West China Broadcasting TV, 2014, 08:121-122.

[2] Yang Liu. Design of intelligent voice home appliance control system based on ZigBee[J].Hunan University of Science \& Technology,2015.

[3] Peihe Wang. Research on the trigger method of taxi emergency call system based on speech recognition [J]. Ocean University of China, 2014.

[4] Dong Yu, Li Deng. Analytical depth learning: Practice of speech recognition [M]. Beijing: Electronics industry Press, 2016.

[5] Yi Zhang. Speech processing and human computer interaction technology [M].Beijing: Science Press, 2016.

[6] (United States)Lawrence R Rabiner, Ronald W Schafer. Theory and application of digital speech processing[M].Beijing: Electronics industry Press,2016.

[7] Lin Bai. Research on robot control technology based on speech recognition [D]. Southwest Petroleum University, 2014.

[8] Liang Min. Research and implementation of speech recognition technology for smart home [D]. Harbin Industrial University, 2013.

[9] Minxu Wang. Research and development of speech recognition technology [J].Microcomputer \& Its Applications, 2009, 23:1-2+6.

[10] Ying Zhou. Analysis on the development trend of speech recognition technology [J].Computer CD software and Application, 2012, 19:141-142.

\section{Brief Introduction of the Writer}

Zhuqingsong, male, was born in 1973 in Jiangsu Peixian of China, associate professor, Ph. D candidate, graduated from China University of Mining and Technology(Beijing) and obtained a master's degree of mechanical electronic engineering in 2004, Mainly engaged in mechanical and electrical equipment research, and now is a teacher of Beijing Polytechnic, published many academic papers in recent years. 